\title{
Using APASS and 2GSS for studying variable stars
}

\author{
Arne Henden ${ }^{1, \star}$, Stephen Levine ${ }^{2}$, Doug Welch ${ }^{3}$, Dirk Terrell ${ }^{4}$, Ulisse Munari ${ }^{5}$, and \\ Thomas Smith ${ }^{5}$ \\ ${ }^{1}$ American Association of Variable Star Observers, Cambridge, MA, USA \\ ${ }^{2}$ Lowell Observatory, Flagstaff, AZ, USA \\ ${ }^{3}$ McMaster University, Hamilton, ON, Canada \\ ${ }^{4}$ Southwest Research Institute, Boulder, CO, USA \\ ${ }^{5}$ Dark Ridge Observatory (DRO), Weed, NM, USA
}

\begin{abstract}
The AAVSO Photometric All-Sky Survey (APASS) provides calibrated magnitudes in the range $7<V<17$ for the entire sky, in the $B V u g r i Z_{s} Y$ bandpasses. While primarily designed for 0.02 mag calibration, it provides photometry over a many-year baseline, and also has near daily cadence in the standard field regions on the equator. Likewise, the Second Generation Synoptic Survey (2GSS) is a follow-on to APASS, and will provide daily cadence in two passbands for the entire sky.
\end{abstract}

\section{Introduction}

APASS was begun in 2010 in response to the need for accurate, bright, photometric all-sky calibration. The intent was to extend the Sloan Digital Sky Survey calibration to brighter magnitudes, as well as provide transitional photometry in both the Johnson $(B, V)$ and Sloan (ugriz) passbands. This survey has had ten data releases, and covers the entire sky from $7<V<17$ mag. The photometry is accurate to about $0.02 \mathrm{mag}$, and the astrometry is accurate to about 0.15 arcsec.

Data collection is accomplished with telescope systems at CTIO in the south and DRO in the north. Each telescope system uses a single Paramount ME mount, with two bore-sighted optical telescope assemblies (OTAs). One OTA covers the blue photometric bands $\left(B, u, g, Z_{s}, Y\right)$ while the other OTA simultaneously covers the mid-range photometric bands $(V, r, i)$. The OTAs are $20 \mathrm{~cm} \mathrm{f} / 3.6$ ASA astrographs, along with Apogee U16m CCD cameras. Each OTA covers a $2.9 \times 2.9$ degree field of view, with 2.5 arcsec pixels. The survey is designed to cover the entire sky twice with a small amount of overlap between each telescope field. Because there is vignetting in the system and poor image correction in the field corners, a set of secondary field centers, offset from the primary by center-to-corner, is also taken twice. Therefore, the final survey has a minimum of four observations of each object. In reality, many more observations are made, due to non-photometric nights and other processing issues. In addition, nightly standard-star fields are acquired, with several hundred observations for each over the 6-year timespan of the survey. Further information about the survey can be found at https://www . aavso.org/apass/.

^arne@aavso.org 
The SHARCnet system of high performance computers (https://www. sharcnet.ca/my/front/) is used to find and measure all objects using a combination of Sextractor, DAOPHOT and astrometry.net software. All measures from photometric nights are combined into a master "means" catalog, which is available from the AAVSO or VizieR. We also take all individual measurements with their Julian Date and import them into an Epoch Photometry Database (EPD) that is available to AAVSO members.

\section{Variable stars}

APASS has been used for variable star studies in five ways. The obvious first use is in providing calibrated secondary comparison stars in the fields of known variables. This was one of the primary reasons for the development of the survey. The means catalog is heavily used by the AAVSO in creating their charts. Second, on non-photometric nights, specific target fields have been observed. These are targets such as recent novae, Kepler fields, comets, or fields relating to specific research projects. Third, we also used a couple of dozen nights for a study of the Aquarius Halo Stream ([1]), re-imaging each field often enough to search for RR Lyr variables. Fourth, several researchers have queried the photometric database for stars that lie in a specific locus of color-color space, to be used as a target list for studying a specific class of variable star (such as cataclysmic variables). Finally, the EPD itself contains many fields for which some known variable stars exist, and for which other variables can be discovered (or analysis made if other researchers publish new discoveries).

\section{2GSS - the APASS follow-on}

Once the APASS survey is complete, we hope to be able to use the hardware to continue observing. The intent is to keep nearly everything the same, except to replace the $20 \mathrm{~cm}$ astrographs with 30 $40 \mathrm{~cm}$ astrographs, and to only image in one filter per OTA (Sloan $g$ and $i$ ). This survey technique will optimize both the simultaneous wavelength coverage and the number of fields that can be observed per night. With five such systems, we envision nightly coverage of the available sky. Since the APASS means catalog will be available, no standard fields need to be observed, and each target field will have sufficient secondary standards to zero-point the photometric measures. With sufficient computing power, it should be possible to instrumentally calibrate the images and extract stellar photometry in near-real-time, and provide alerts for those objects exceeding a specified photometric variation. This is similar in many ways to the envisioned use of LSST, with two exceptions: we will have simultaneous 2-color photometry, and the faint limit of 2GSS overlaps slightly with the bright limit of LSST, providing continuous temporal coverage of the sky from 7th magnitude to the LSST limit. A pilot 2GSS system is currently operational at Lowell Observatory.

Acknowledgments: This project was begun with a grant from the Robert Martin Ayers Sciences Fund. APASS is funded in part through NSF grant AST-1412587. We appreciate the help provided by: J. Irwin (Harvard/MEarth) and D. Reichart (UNC); vendors including Software Bisque, DC3 Dreams, Apogee, Astrodon and ASA; and dozens of volunteers.

\section{References}

[1] Funari, U., Henden, A., \& Frigo, A., NewA, 27, 1 (2014) 